\title{
Cannabinoides y conducta adictiva
}

\author{
Navarro, M.; Rodriguez de Fonseca, F. \\ Departamento de Psicobiología. Instituto Universitario de Drogodependencias
}

Dirigir correspondencia: Miguel Navarro. Departamento de Psicobiología. Instituto Universitario de Drogodependencias. Facultad de Psicología. Universidad Complutense de Madrid. 28223 Madrid.

\begin{abstract}
Resumen
Los cannabinoides son compuestos psicoactivos presentes en el cannabis y que actúan en el sistema nervioso a través de receptores específicos de membrana, los receptores CB-1. Estos receptores están situados en neuronas de muchos circuitos encefálicos, incluyendo el sistema de recompensa cerebral. Este sistema es clave para entender la conducta adictiva, y de él forman parte las neuronas dopaminérgicas mesotelencefálicas, así como algunas neuronas peptidérgicas de entre las que destacan las neuronas encefalinérgicas. Los cannabinoides, al igual que el resto de las drogas de abuso, activan las neuronas mesotelencefálicas y disminuyen el umbral de recompensa cerebral. Del mismo modo que la cocaína, los opiáceos o el etanol, estos compuestos inducen conductas de autoadministración en animales de experimentación y provocan condicionamiento de lugar preferencial. La administración crónica de cannabinoides provoca tolerancia y dependencia, e induce neuroadaptaciones en el circuito de la recompensa que son idénticas a las inducidas por la principales drogas de abuso y que se pueden poner de manifiesto mediante el cese de la administración de estos compuestos (síndrome de abstinencia comportamental y bioquímico específico). Los cannabinoides actúan sinérgicamente con el sistema opioide endógeno, en especial con el sistema encefalinas-receptor-F -opioide, lo que les permite actuar como factores de vulnerabilidad en el desarrollo de la conducta adictiva. La existencia de una interacción opioide-cannabinoide permitirá abrir nuevas puertas terapéuticas para la adicción a heroína y a etanol.
\end{abstract}

Palabras clave: cannabis, sistema cannabinoide, adicción, dopamina, sistema de recompensa, modelos animales, opiaceos, cerebro, dependencia.

\section{Summary}

Cannabinoids are the psicoactive compounds found in cannabis preparations. They act through the activation of specific membrane receptors termed cannabinoid CB-1 receptors. These receptors are located in neurons of multiple encephalic circuits, including the reward system. This reward system is essential for the outcome of the addictive behavior, and it is composed of several circuits including the mesotelencephalic dopaminergic system and telencephalic peptidergic neurons such as enkephalinergic cells .Cannabinoids activate mesotelencephalic dopaminergic neurons and lower intracranial self stimulation thresholds, as do major abused drugs. Like cocaine, ethanol or the opiates, cannabinoids induce conditioned place preference and are self-administered by laboratory rodents. Chronic administration of cannabinoids results in tolerance and dependence, as well as in neuroadaptions in brain reward circuits that resemble those found for major drugs of abuse. A specific biochemical and behavioural withdrawal syndrome can be observed in cannabinoid-dependent animals. Cannbinoids act sinergically with endogenous opioid peptides, specially with the enkephalin-F-opioid system.

This interaction could even help to open new therapeutic doors to heroin and alcohol addiction.

Key words: cannabis, cannabinoid system, addiction, dopamine, reward system, animal models, opiods, brain, dependence. 


\section{L} a utilización médica de las preparaciones de la planta cannabis sativa (hashish, marihuana y bhang) se ha podido constatar que data desde hace 4000 años en documentos de la medicina asiática, aunque su uso recreacional y hedonista sea probablemente anterior. En este sentido, hay desde las descripciones realizadas por Heródoto del uso ritual del cannabis por los escitas, pueblo asiático muy guerrero del s. VII a. de J.C., a las de aquellos caballeros medievales creados por el viejo de la montaña (los hashashins) o las imágenes de los conciertos de rock de los años 60, en las que siempre estaba presente el consumo masivo de esta droga.

Desde las mil y una noches hasta los cuentos de Cornell Woolrich, la literatura tiene presente los efectos distorsionantes del cannabis sobre la percepción, la emoción, la memoria y el peculiar mito del estímulo sobre la creatividad artística.

A partir del aislamiento y síntesis en 1964 del $\Delta^{9}$-tetrahidrocannabinol (THC), constituyente psicoactivo principal de la planta, realizado por Gaoni y Mechoulam hasta finales de los ochenta, la base neurofarmacológica del THC ha constituido un misterio y un desafío para la ciencia y todavía en la actualidad no se conoce qué dimensión neurocientífica pueden suponer los avances realizados en estas dos últimas décadas sobre la transmisión cannabinoide (Tabla 1).

La característica farmacológica de los cannabinoides, a diferencia de otros fármacos, no viene definida por un efecto particular sino más bien por un perfil farmacológico que consta de la tétrada: analgesia, catalepsia, hipotermia y alteración de la actividad locomotora. Sin embargo, todavía las propiedades reforzadoras de los cannabinoides psicoactivos permanecen sujetas a un intenso debate social y científico, hasta conocer con mejor detalle qué propiedad reforzadora propia poseen y qué mecanismo neurobiológico comparten con el resto de las drogas de abuso.

Ese interés deriva de dos aspectos principales: En primer lugar, porque las preparaciones de cannabis sativa siguen siendo las más consumidas en el mundo con respecto al resto de drogas de abuso ilegales; y en segundo lugar, porque actúan a través de un sistema propio de señalización neuronal muy ubicuo en el SNC y fuera de él, con importantes implicaciones neurobiológicas y psicobiológicas; por lo que su mejor compresión arrojará luz en la etiopatogenia de algunas enfermedades neuropsiquiátricas, entre las que una de ellas, bien puede ser la conducta adictiva.

\begin{tabular}{|c|c|}
\hline \multicolumn{2}{|c|}{$\begin{array}{l}\text { Tabla 1. PRINCIPALES AVANCES EN LA INVESTIGACIÓN RELACIONADA } \\
\text { CON EL SISTEMA CANNÁBICO ENCEFÁLICO } \\
\text { (referencias bibliográficas en el monográfico Neurobiology of Disease Experimental Neurology, 5(6), diciembre 1998). }\end{array}$} \\
\hline EVENTO & FECHA \\
\hline USO MÉDICO DEL CANNABIS & $\begin{array}{l}3000 \text { años a J.C. en la medicina } \\
\text { tradicional asiática }\end{array}$ \\
\hline AISLAMIENTO PRINCIPIOS PSICOACTIVOS & 1964 \\
\hline CANNABINOIDES SINTÉTICOS & 1980-1988 \\
\hline IDENTIFICACIÓN DEL RECEPTOR CB ${ }_{1}$ & 1988 \\
\hline CARTOGRAFÍA CEREBRAL CB ${ }_{1}$ & 1990 \\
\hline CLONAJE DEL RECEPTOR CB & 1990 \\
\hline NEUROFARMACOLOGÍA DEL CB 1 & 1988-1995 \\
\hline AISLAMIENTO Y BIOQUÍMICA ANANDAMIDA & 1992-1995 \\
\hline NEUROFARMACOLOGÍA ANANDAMIDA & 1993-1997 \\
\hline SIINTESIS ANTAGONISTA SR-141716A & 1994 \\
\hline AISLAMIENTO Y FUNCIÓN DEL 2-Ara-G & 1995-1997 \\
\hline NEUROANATOMÍA FUNCIONAL DEL CB ${ }_{1}$ & 1996-1997 \\
\hline SISTEMA DE RECAPTACIÓN ANANDAMIDA & 1997 \\
\hline
\end{tabular}




\section{REFUERZOY RECOMPENSA}

Todos los mamíferos necesitan conseguir una variedad de elementos situados en el entorno para sobrevivir, de modo que a medida que se va conociendo el valor de cada estímulo (alimento, información, sexo, etc...) como factor de supervivencia aumenta la aproximación a él y su consumo (reforzadores). Esa capacidad se ha ido adquirido evolutivamente y explica porqué los reforzadores son factores motivacionales principales, que seleccionan e inician los actos voluntarios orientando y gobernando la conducta para alcanzar aquellos objetivos establecidos voluntariamente.

La selección de un refuerzo se elabora a partir de opciones comportamentales, en virtud a tres factores: el valor motivacional del refuerzo, que depende de la cualidad, magnitud y probabilidad con la que el refuerzo vuelva a aparecer, del estado emocional o motivacional que posea el individuo y de la presencia de alternativas disponibles. En este caso, cuando existen varias opciones que compiten entre sí, puede predecirse la opción elegida en función a las expectativas que genera cada una de ellas en el individuo (Schultz et al., 1997).

El deseo de un refuerzo puede representar un indicador de afecto o preferencia (refuerzo positivo), que en el caso del consumo de drogas de abuso lo representa la búsqueda de un sabor, de efectos euforizantes, o sensaciones subjetivas agradables una vez consumida. Los consumidores de cannabis reflejan un incremento de la sensación de bienestar, euforia moderada, relajación y alivio de la ansiedad, pocas personas que fuman socialmente marihuana buscan intensificar las emociones, la distorsión sensorial, alucinaciones moderadas o la ansiedad, que son propias del consumo de dosis elevadas (Abood y Martin, 1992).

También el consumo de haschish se hace para evitar el estado aversivo por las consecuencias del consumo prolongado o para mitigar trastornos preexistentes de la personalidad o del temperamento, que represen- tan el refuerzo negativo, que también aumenta la probabilidad de repetir la autoadministración, incrementando la expectativa de terminar o disminuir la condición desagradable. Los refuerzos inducen estados emocionales subjetivos, que cuando son placenteros se les denomina recompensa.

Desde el trabajo pionero de Olds y Milner en 1954 con el descubrimiento en primates de los circuitos cerebrales del refuerzo y la recompensa, hay una gran cantidad de investigaciones relacionadas con estos fenómenos y sus implicaciones neurobiológicas y psicobiológicas. La recompensa contribuye al aprendizaje y mantenimiento de las conductas preparatoria (de acercamiento) y consumatoria, que sirven para seleccionar y conseguir los componentes más importantes del entorno y contribuyen a que la supervivencia y adaptación sean más eficientes.

Los efectos recreacionales y la predisposición al consumo abusivo de las drogas derivan de las propiedades de aumentar la activación de los mecanismos reforzadores cerebrales y parece ser la propiedad común y esencial de todas ellas, produciendo efectos subjetivos que constituyen, según la jerga, el high, rush o subidón que deriva de la capacidad de las drogas de abuso de inducir refuerzo positivo y es la consecuencia de actuar directa e indirectamente sobre los citados circuitos, donde la distribución de los receptores cannabinoides (CB1) coincide en aquellas áreas directamente implicadas en el circuito de la recompensa cerebral (Henkerham, 1992).

\section{LOS CANNABINOIDES INTERACCIONAN CON EL SUBSTRATO NEUROBIOLÓGICO DEL REFUERZO POSITIVO}

Mediante numerosos estudios neurofarmacológicos, se ha establecido la implicación del sistema dopaminérgico en los efectos reforzadores de las drogas de abuso. La magnitud de la recompensa muestra una relación estrecha con el grado de activación de la vía dopaminérgica ascendente mesolímbicacortical, originada en el área tegmental ventral, y 
que proyecta a estructuras corticales anteriores y subcorticales del sistema límbico (Corbett y Wise, 1980).

Existen múltiples evidencias que ponen de manifiesto que los mecanismos responsables de la recompensa dependen de la integridad funcional del citado sistema dopaminérgico y de sus conexiones (Fray et al., 1983) y su activación está íntimamente asociada con los efectos reforzadores positivos de las drogas de abuso, puesto de manifiesto mediante muchos paradigmas comportamentales que evaluan las propiedades reforzadoras positivas de las diferentes drogas de abuso (Gardner, 1997).

Éste sistema esta compuesto por dos proyecciones principales: La vía nigroestriatal, que desde la pars compacta de la substancia nigra proyecta al núcleo caudado de los ganglios basales y la vía mesolímbicacortical que desde el área tegmental ventral proyecta al núcleo accumbens, el tubérculo olfatorio, el córtex frontal y amígdala. Este último circuito, es el que primariamente está implicado en las acciones reforzadoras positivas de las drogas de abuso (Corbett y Wise, 1980).

La vía mesolímbicacortical es la más sensible a los efectos agudos de los cannabinoides, como demostró el grupo de E. Gardner por el aumento del flujo dopaminérgico en el núcleo accumbens tras la administración de dosis bajas de THC, y también, mediante registros extracelulares directos se ha podido establecer que tras la administración de agonistas CB1 se incrementa la actividad de las neuronas dopaminérgicas del área tegmental ventral (French, 1997), unido a un aumento de la liberación de dopamina en otras áreas de proyección mesolímbicas (Chen et al., 1990, Gardner y Vorel, 1998). Sabemos que las drogas de abuso tienen propiedades reforzadoras o de recompensa, aunque se presta poca atención a los mecanismos psicobiológicos subyacentes a dichos efectos.

Las propiedades reguladoras de la transmisión dopaminérgica frente a los reforzadores, ya sean estímulos naturales (alimentos, estímulos sexuales, etc...) o drogas de abuso, dependen de qué porción se active del núcleo accumbens, cuya división consta de la porción central o core y la más ventromedial o shell.

El núcleo accumbens ofrece cambios precisos en la transmisión dopaminérgica en la porción del shell y del core en respuesta a estímulos apetitivos o aversivos. De manera que aquellos estímulos apetitivos inusuales, imprevistos o incondicionados provocan una respuesta importante en el shell, mientras que la respuesta es muy pobre cuando el estímulo es aversivo o si cualquiera de ellos es habitual o previsto.

Sin embargo, el core responde a estímulos motivacionales genéricos o aversivos e incluso la respuesta puede sensibilizarse si el estímulo aversivo se repite (Salamone et al., 1999).

Estas diferencias funcionales del núcleo accumbens sugieren que la respuesta adaptativa dopaminérgica del shell se produce esencialmente frente a la adquisición (aprendizaje); mientras que, el core responde a la expresión de la motivación (respuesta).

A este respecto, no existe una idea concreta del lugar donde los cannabinoides estimulan los receptores CB1 situados en las neuronas dopaminérgicas, ni qué papel representan los receptores D1 y D2 en la respuesta por la estimulación de la vía mesolímbicacortical ejercidad por los cannabinoides; aunque se ha puesto de manifiesto que tras la administración intravenosa de cannabinoides existe un incremento notable de dopamina en ambas porciones del núcleo accumbens, más intensamente en el shell al igual que sucede con otras drogas de abuso (Tanda et al., 1997)

Esta respuesta no se afecta bloqueando los receptores CB1 con el antagonista SR141716A en el área tegmental ventral (Gueudet et al., 1995), mientras que si lo hace con antagonistas del receptor $\mu$ (naloxona, naloxonacina), evitándose la liberación de dopamina, muy probablemente sea debido a través de las neuronas del área tegmental ventral (Tanda et al., 1997), aunque también pudiera ser en las terminales que proyecta al núcleo accumbens (Gardner y Vorel, 1998), o también cabe la posibilidad que actúe por estimulación transináptica. 
Por otra parte, resulta interesante destacar la asociación entre las acciones de los cannabinoides y la respuesta al estrés. Como antes hemos indicado, los agonistas cannabinoides activan la vía mesolímbicacortical, y para ello dependen de la respuesta adrenal liberando glucocorticoide. Dado que el estrés agudo se asocia con una activación mesolímbicacortical mediada por receptores glucocorticoideos presentes en neuronas dopaminérgicas de la citada vía (Piazza et al., 1996), puede entenderse que los canabinoides son equivalentes a estresores químicos puesto que activan el eje adrenal a través de la liberación de ACTH (Martín-Calderón, et al., 1998), induciendo una respuesta comportamental similar al estrés (Rodríguez de Fonseca et al., 1996), ya que el núcleo central de la amígdala y el núcleo paraventricular del hipotálamo, que participan íntimamente en esta respuesta emocional, presentan ambos receptores CB1 (Navarro et al., 1997).

Teniendo en cuenta que la actividad dopaminérgica mesoaccubal puede organizarse a modo de una cadena fisiopatológica, y da base a la vulnerabilidad a las drogas de abuso (Piazza y Le Moal, 1996), y si la activación mesolímbicacortical inducida por cannabinoides depende de la activación del eje adrenal, puede dar pie al soporte epidemiológico de que el consumo de cannabis es un factor de vulnerabilidad a los efectos adictivos de las drogas de abuso o a la propensión de la formación de hábitos tóxicos (Gardner y Vorel, 1997); así como el hecho de aquellas observaciones clínicas que establecen que el consumo de cannabis provoca una predisposición al inicio de enfermedades neuropsiquiátricas, como la psicosis (Andreasson et al., 1987; Emrich et al., 1997).

\section{CANNABINOIDES Y PARADIGMAS COM- PORTAMENTALES ADICTIVOS}

Los modelos animales evalúan las propiedades reforzadoras que tienen las drogas de abuso y permiten comparar la capacidad adictiva de las mismas, estableciendo grados entre los posibles factores que contribuyen en el refuerzo, la dependencia, el síndrome de abstinencia y la recaída, y también facilitan la identificación de aquellos mecanismos neurobiológicos que intervienen en los citados fenómenos desde el nivel molecular al comportamental. En este sentido, los cannabinoides, como el resto de las drogas de abuso, comparten una serie de características comunes, que analizaremos a continuación.

Primero, aumentan la recompensa inducida bajo el paradigma conocido como autoestimulación eléctrica intracraneal (AEIC), a los animales entrenados a presionar una palanca en una caja operante, se les implanta electrodos en el haz prosencefálico medial y son capaces de autoestimularse pequeñas descargas eléctricas al activar la palanca, de modo que este paradigma permite conocer el umbral de recompensa cerebral que tiene un animal en cada situación experimental.

En 1988 el grupo de Elliot Gardner demostraron con este paradigma, que las ratas Lewis eran las más sensibles al efecto reforzador del THC a dosis bajas, de manera que la administración intravenosa de $1,5 \mathrm{mg} / \mathrm{Kg}$ de peso de $\mathrm{THC}$, disminuye el citado umbral, como ocurre con otras drogas de abuso, como el etanol, heroína, cocaína y nicotina, que también disminuyen el citado umbral tanto más cuanto más refuerzo positivo poseen (Gardner y Lowinson, 1991); asímismo, y complementariamente, la administración de $1.0 \mathrm{mg} / \mathrm{Kg}$ de THC, disminuye la frecuencia de estimulación eléctrica intracraneal, que como el anterior, es otro índice adictivo (Lepore et al., 1996).

En segundo lugar, está ampliamente constatado por numerosos equipos de investigación que la administración aguda de agonistas del receptor CB1 aumenta la activación basal de las neuronas dopaminérgicas de aquellos circuitos relacionados con el refuerzo y la recompensa, y también lo hace la síntesis, la liberación basal o la inhibición de la recaptación dopaminérgicas (Poddar y Dewey, 1980; Navarro et al., 1993 y Tanda et al., 1997). Particularmente aumenta la activación dopaminérgica en la porción del shell del núcleo accumbens, donde parece probable que esta ruta neuroquímica actúe como un filtro de entrada 
de las señales nerviosas generadas en el sistema límbico, mediando los aspectos motivacionales que conducirían a que los estímulos asociados a su consumo cobren un significado emocional especial del que antes carecían, facilitando la formación de hábitos tóxicos (Gardner y Vorel, 1998). Estos efectos son ampliamente reconocidos como lo más característico de las acciones agudas de las principales drogas adictivas (Koob et al., 1998).

En tercer lugar, bajo el paradigma de autoadministración intravenosa que es un paradigma clásico para la evaluación de la capacidad adictiva de las drogas de abuso, el grupo de Walter Fratta ha conseguido que ratones se autoadministren un potente agonista cannabinoide, el de WIN-55,212-2 $(0.1 \mathrm{mg} / \mathrm{Kg})$, mientras que ratones transgénicos, que carecen genéticamente del receptor CB1, bajo idénticas condiciones experimentales no son capaces de autoadministrarse la droga y carecer de aquellos efectos farmacológicos relacionados con el efecto adictivo de las acciones agudas o crónicas de la misma (Ledent et al., 1999). Esto supone una mediación de los efectos reforzadores de los cannabinoides a través del receptor $\mathrm{CB}$ 1. El hecho de que el THC no haya podido generar este mismo paradigma en otras especies, como lo hacen la nicotina, etanol, heroína o cocaína; puede obedecer probablemente a razones farmacocinéticas entre el WIN-55,212-2 y otros cannabinoides (Martellota, et al., 1998), más que a razones de tipo farmacodinámicas.

En cuarto lugar, los cannabinoides pueden inducir condicionamiento de lugar preferencial (CLP), un modelo animal en el que el estímulo que representa el entorno donde se le administra la droga, se asocia a los efectos de búsqueda o deseo de la misma. En numerosos estudios se ha demostrado que los estímulos neutros asociados a otros contingentes (autoadministración intracraneal o intravenosa) o con los no contingentes (experiencia de sensaciones subjetivas del medio externo o interno) adquieren propiedades reforzadoras similares a las que tienen las drogas de abuso primariamente.
Hasta hace poco no se había conseguido evaluar que los cannabinoides tuvieran esa capacidad, sin embargo, parece que el THC tiene efecto apetitivo pero muy dependiente de la dosis administrada, de modo que con 1 $\mathrm{mg} / \mathrm{Kg}$ produce un efecto CLP potente, mientras que por el contrario, entre las dosis 2 a 4 $\mathrm{mg} / \mathrm{Kg}$ induce aversión (Lepore et al., 1995); de manera similar, el efecto del THC es dependiente del tiempo que transcurre desde el emparejamiento con el entorno y el consumo de THC. En humanos sucede un fenómeno paralelo, en el que el tiempo y la dosis son factores determinantes para que el THC tenga efecto condicionante de lugar o aversivo (Noyes et al., 1975, Raft et al., 1977 y Laszlo et al, 1981).

Desde una amplia variedad de datos y paradigmas, con independencia de los diferentes grupos de investigación, parece claro que los cannabinoides activan los circuitos implicados en el refuerzo/recompensa de los mamíferos bajo diferentes parámetros electrofisiológicos, neuroquímicos o comportamentales de modo no diferente al resto de drogas de abuso, aunque durante un tiempo se les ha considerado como drogas de abuso atípicas por no haber podido demostrar hasta el momento que el THC tiene propiedades similares al resto. Es probable que las dificultades en la demostración del incumplimiento del THC de los mismos criterios adictivos que otras drogas de abuso, sean debidas a varios factores, entre los que pueden estar los efectos motores depresores, los componentes aversivos, las características farmacocinéticas derivadas de su liposolubilidad y la falta de modelos experimentales.

\section{CANNABINOIDES Y DEPENDENCIA}

¿Los cannabinoides son capaces de inducir efectos crónicos similares a los que provocan el resto de las principales drogas de abuso? La investigación puede responder afirmativativamente si cumplen una serie de requisitos, entre los cuales tenemos los siguientes: En 
primer lugar, los cannabinoides son capaces de inducir tolerancia a los principales efectos farmacológicos en la mayoría de especies y también en el humano. Aunque no se presenta por igual a todos los efectos, existe una tolerancia cruzada entre los distintos grupos de cannabinoides, pero es mucho más prolongada que la observada frente a otras drogas de abuso, como la inducida por los opioides (Dewey, 1986).

En segundo lugar, los cannabinoides inducen dependencia, evidenciada por el grupo dirigido por Michael Walker en 1995, manifestando un conjunto de síntomas similares a los observados durante el síndrome de abstinencia a opioides, como son: la sacudida corporal (wet-dog shake) el autoacicalamiento (self-grooming) tras la administración de un antagonista cannabinoide, después de administración crónica de $15 \mathrm{mg} / \mathrm{Kg}$ i.p. de THC. El síndrome de abstinencia, aparece como un conjunto de síntomas y signos, caracterizados por breves secuencias motoras frecuentemente interrumpidas antes de alcanzar el final de las mismas en las que se incluyen síntomas como frotamiento facial con las patas delanteras y temblor de estas, secuencias de rascado, vocalizaciones, etc...; y otros síntomas como ptosis palpebral, piloerección, diarrea, hipertermia y postura corporal anómala, no observándose pérdida de peso (Tsou et al., 1995).

Posteriormente, otros autores han podido ratificar esos resultados (Aceto et al., 1995) en otras especies (Fredericks \& Benowitz, 1980, Beardlsley et al., 1986 y Hutcheson et al., 1998).

En tercer lugar, a los síntomas comportamentales específicos se asocia un conjunto de fenómenos neuroquímicos y neuroendocrinos similares a lo sucedido con otras drogas de abuso, ya que tras la exposición crónica con otros agonistas cannábico muy selectivo del receptor CB1 una vez administrado el antagonista SR141716A se experimenta la misma manifestación comportamental con un curso temporal similar al incremento de los niveles extracelulares del CRF en la amígdala cerebral, medidos in vivo, mediante microdiálisis cerebral (Rodríguez de Fonseca et al., 1997) en el que participan también otras estructuras límbicas puesto de manifiesto por el incremento de la inmunoreactividad Fos en áreas corticales (hipocampo y piriforme), tronco cerebral (ATV, locus coeruleus, núcleo del tracto solitario, substancia nigra, núcleos pericacueductales), hipotálamo (núcleo paraventricular, supraquiasmático, supraóptico), núcleo anterior paraventricular del tálamo y los ganglios basales; todas las estructuras implicadas en los mecanismos neurobiológicos del estrés. Estas consecuencias, son similares a las acontecidas durante el síndrome de abstinencia a etanol, cocaína y opiáceos y explican, en parte, el estado emocional común de los efectos cerebrales de la dependencia (Koob, et al, 1998).

$Y$ en cuarto lugar, se ha podido implicar el sistema dopaminérgico mesolímbicocortical en las consecuencias aversivas del síndrome de abstinencia a cannabinoides en ratas, produciéndose cambios plásticos en las neuronas dopaminérgicas situadas en el ATV que proyectan al núcleo accumbens, este fenómeno, se ha podido poner de manifiesto mediante la reducción de la actividad electrofisiológica en las citadas neuronas (Diana et al., 1998).Curiosamente, similares cambios ocurren en el síndrome de abstinencia al etanol (Diana et al., 1993) y a la morfina (Diana et al., 1999).

Es probable que el fenómeno de la abstinencia no se haya observado hasta contar con un antagonista específico del receptor CB1 y a la redistribución que sufren los cannabinoides debido a su elevada hidrofobicidad que les hace depositarse en el tejido graso y tener una semivida muy prolonga, este detalle farmacocinético impide una caída brusca en los niveles plasmáticos (Dewey et al., 1984), llegando a estar presente el THC en el receptor durante un tiempo mayor incluso que al plasmático (Seth y Sinha, 1991) y evita que espontáneamente aparezca un fenómeno abrupto y severo de abstinencia, a diferencia de lo que sucede con otras drogas de abuso de semivida mucho más corta. 
En el humano, la manifestación de abstinencia tras el consumo crónico, se pone de manifiesto por insomnio, inquietud, anorexia e irritabilidad (Jones et al., 1976), estos síntomas no parecen lo suficientemente alarmantes si se comparan a otras drogas de abuso y en los últimos veinte años han recibido poca atención en la investigación experimental. Sin embargo, estudios epidemiológicos recientes en los E.E.U.U. indican que el $5 \%$ de los estudiantes de institutos la fuman diariamente (Johnston et al., 1997), con una prevalencia estimada de diagnóstico de dependencia que supera al $4 \%$ de la población general (Anthony et al., 1994), de la cual el 7,4\% son adultos y el $14,4 \%$ adolescentes, que cumplen criterios diagnósticos de dependencia durante el año anterior al evaluado; de forma que, el $93 \%$ de los consumidores indican su incapacidad de dejar de fumarla y buscan tratamientos para evitar la dependencia, especialmente cuando se hace publicidad en los medios de comunicación (Haney, et al., 1999).

Estudios recientes, señalan que la sintomatología de la abstinencia va dirigida al aumento de la conducta agresiva inespecífica (Kouri et al., 1999). Aunque, en general queda por estudiar la relevancia clínica del citado fenómeno y las características temporales, los síntomas antes indicados, pueden ser particularmente importantes dependiendo si se asocian a signos de abstinencia a etanol, opiáceos o cocaína pudiendo representar un papel importante en la recaída, ya que se estima que entre un 20-30\% de las recaídas, pueden provenir por el aumento de la vulnerabilidad adictiva (Hall et al., 1994), o participar en el desarrollo de trastornos neuropsiquiátricos, como: esquizofrenia, manía o trastornos relacionados (Castle y Ames, 1996) o efectos neuropsicológicos residuales detectados en pruebas atencionales, verbales y de memoria visoespacial, que persisten una vez abandonado su consumo durante un tiempo prolongado (Pope et al., 1995).

El consumo de cannabinoides a largo plazo es particularmente sensible en los adolescentes durante la pubertad, porque su uso a menudo provoca trastornos comportamenta- les, como depresión, déficit atencional o hiperactividad y dependencia (78\%) y precede al uso de otras de abuso (Crowley, et al., 1998); y aunque, su consumo no sea necesariamente un factor predictor de la progresión al consumo de otras drogas, los adolescentes que consumen marihuana tienen 104 veces más probabilidad en consumir cocaína frente a los que nunca la han consumido (Comité de la Academia Pediátrica Americana, 1999), de manera que el consumo de haschish representa una conducta de riesgo y hay que tenerla muy en cuenta como droga que genera hábitos tóxicos, pudiendo representar un fenómeno de puerta de entrada para algunos adolescentes.

\section{INTERACCION FUNCIONAL ENTRE EL SISTEMA CANNABINOIDE Y OPIOIDE EN LA CONDUCTA ADICTIVA}

Cuando se compara la distribución cerebral de los receptores CB1 se pone de manifiesto un solapamiento con la distribución neuroanatómica de los receptores opioides $\mu$. Ambos sistemas tienen una presencia muy abundante en los circuitos límbico y áreas motoras, especialmente en la vía mesolímbicacortical donde la liberación de dopamina inducida por THC se realiza a través del receptor $\mu$ localizado en el área tegmental ventral (Tanda et al., 1997). Aunque todavía sea desconocido el posible papel que representa el receptor CB1 en la regulación de la conducta adictiva al cannabis o a otras drogas de abuso, existen muchas propiedades farmacológicas y bioquímicas comunes entre los efectos de los cannabinoides y opioides (Hine et al., 1975; Vela et al., 1995 y Manzanares et al., 1999).

Es interesante notar que la participación del receptor CB1 en los efectos farmacológicos de los opioides, sea sobre todo en aquellos que son a largo plazo y no tanto en los agudos, ya que en ratones transgénicos que carecen del citado receptor, se reduce especialmente el síndrome de abstinencia opioide 
(Ledent et al., 1999). En este mismo sentido, pudimos comprobar la existencia de mecanismos confluyentes entre ambos sistemas cannabinoide y opioide, puesto que al adiccionar animales con morfina o con HU-210 o con ambos, aparecía un síndrome de abstinencia inducido por el antagonista cannábico SR141716A o naloxona respectivamente. Demostrándose además la coexpresión de ambos receptores en áreas como el septum, hipocampo, amígdala y el complejo habenular, que podrían participar en los efectos reforzadores negativos del citado síndrome (Navarro et al, 1998). De este posible efecto cruzado entre la acción adictiva de ambas drogas puede desprenderse dos consecuencias, la primera está relacionada con nuevas estrategias terapéuticas para la dependencia opioide a través del receptor CB1; y la segunda, es que el receptor CB1 puede intervenir en la vulnerabilidad adictiva de los opioides (Fig 1). En este sentido, sabemos que animales expuestos a cannabinoides en etapas precoces del desarrollo, desarrollan una mayor vulnerabilidad a los efectos reforzadores de los opioides, tanto bajo los paradigmas que valoran el condicionamiento pavloviano, como es el condicionamiento de lugar preferencial (Rubio et al., 1995) donde resultan más reforzadoras las dosis bajas de THC (1 y 5 mg/Kg) (Rubio et al., 1998), como también bajo modelos animales que valoran el condicionamiento instrumental (Ambrosio, 1999).

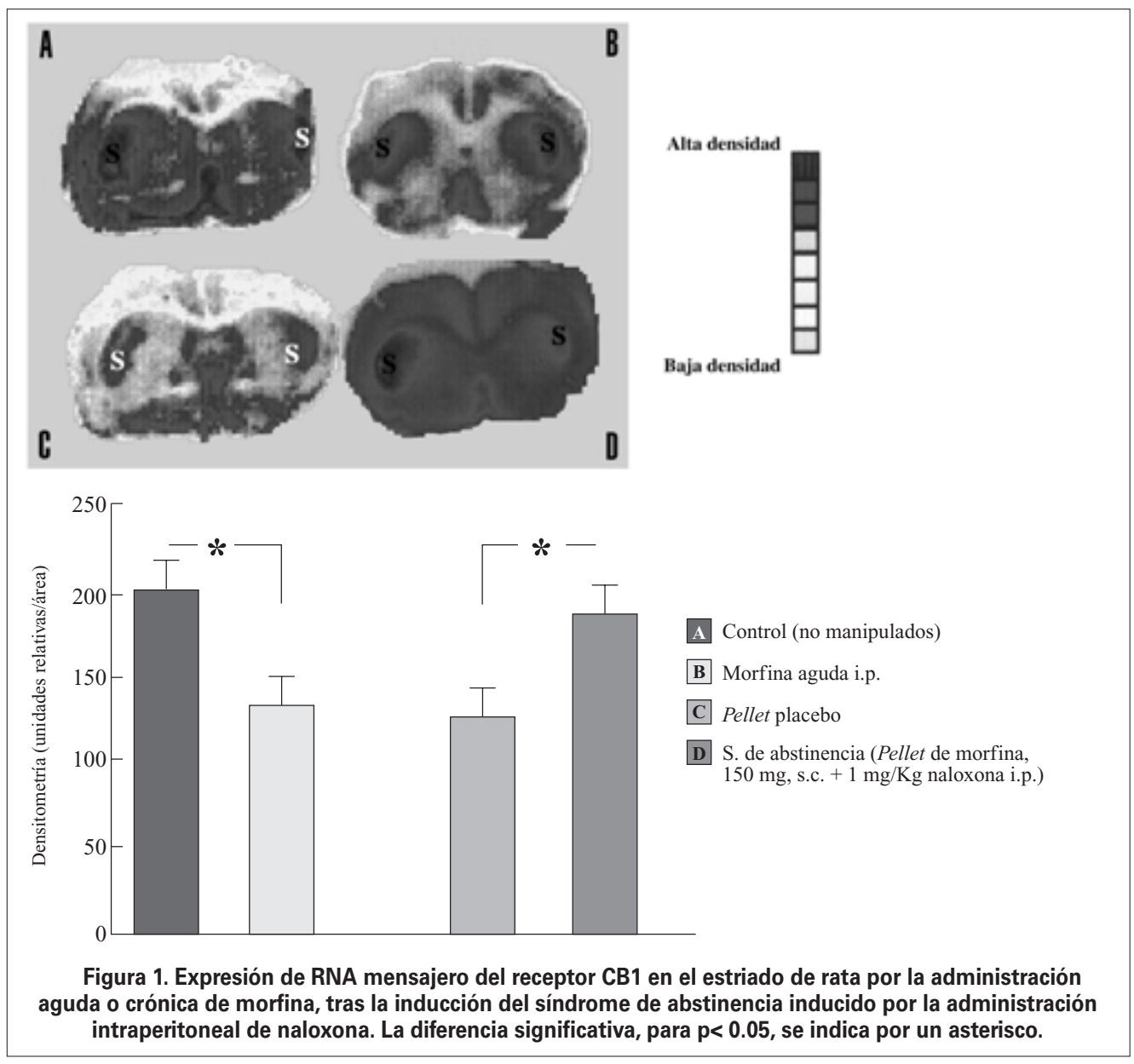


Estos datos, refuerzan la importancia clínica de tener en cuenta las consecuencias a largo plazo del consumo de haschish y abren una ventana conceptual para la comprensión del fenómeno de la dependencia a las drogas de abuso, que en el futuro probablemente proporcione un abordaje terapéutico.

\section{AGRADECIMIENTOS}

Este trabajo está financiado por los siguientes Organismos: Ministerio de Educación y Cultura DGICYT (PM 96/0047), La Delegación del Gobierno para el Plan Nacional Sobre Drogas, La Comunidad Autónoma de Madrid (05/0024/99) y el Hospital Psiquiátrico de Ciempozuelos de la Orden de San Juan de Dios. Los autores quieren agradecer el apoyo concedido por los citados Organismos y también muy especialmente la colaboración de los Dres. Luis Miguel García Segura y Julie Chowen del Instituto Cajal, CSIC, Madrid, por su valiosa asistencia técnica.

\section{REFERENCIAS BIBLIOGRÁFICAS}

Abood, M.E. y Martin, B.R. (1992) Neurobiology of Marijuana Abuse. T.I.P.S., 13: 201.

Aceto, M.D., Scates, S.M., Lowe, J.A. \& Martin, B.R. (1995) Cannabinoid precipitated withdrawal by the selective cannabinoid receptor antagonist, SR 141716A. Eur. J. Pharmacol., 282: R1-R2.

Ambrosio, E., Martin, S., García-Lecumberri, C \& Crespo, J.A. (1999) The neurobiology of cannabinoid dependence: Sex differences and potential interactions between cannabinoid and opioid systems. Life Sci., 65(6-6): 687-94.

American Academy of Pediatrics Committee on Substance Abuse (1999) Marijuana: A continuing Continuing Concern for Pediatricians. Pediatrics, 104(4): 982-985.

Andreàsson, S., Allebeck, P., Engström, A. y Rydberg, V. (1987) Cannabis and Schizophrenia: A longitudinaal study of Swedish conscripts., Lancet, ii: 1483-1486.
Anthony J.C., Warner, L.A., Kessler, R.C. (1994) Comparative epidemiology of dependence on tobacco, alcohol, controlled substances, and inhalant: basic findings from the national comorbidity survey. Exp. Clin. Psychopharmacol., 2: 244-268.

Beardsley, P.M., Balster, R.L. \& Harris, L.S. (1986) Dependence on thtrahydrocannabinol in rhesus monkeys. J. Pharmacol. Exp. Ther., 239: 311319.

Castle, D.J. \& Ames, F.R. (1996) Cannabis and the brain. Austr. New Zeal.. J. Psych., 30: 179-183.

Chen, J., Paredes, W., Lowinson, J.H. y Gardner, E.L. (1990) $\Delta^{9}$-Tetrahydrocannabinol enhances presynaptic dopamine efflux in medial prefrontal cortex. Eur. J. Pharmacol., 190: 259-262.

Corbett, D. y Wise, R.A. (1980): Intracraneal self-stimulation in relation to the ascending dopaminergic systems of the midbrain: a moveable electrode mapping study. Brain Res, 185:1.

Crowley, T.J., Macdonald, M.J., Whitmore, E.A. \& Mikulich, S.K. (1998) Cannabis dependence, withdrawal, and reinforcing effects among adolescents with conduct symptoms and substance use disorders. Drug Alcohol Depend., 50(1): 27-37.

Dewey, W.L. (1986) Cannabinoid Pharmacology. Pharmacol. Rev., 38(2): 151-178.

Diana, M., Melis, M., Muntoni, A.L. \& Gessa, G.L. (1998) Mesolimbic dopaminergic decline after cannabinoid withdrawal. Proc. Natl-Acad. Sci., 95: 10269-10273.

Diana, M., Muntoni, A.L., Pistis, M., Melis, Miriam \& Gessa, G.L. (1999) Lasting reduction in mesolimbic dopamine neuronal activity after morphine withdrawal. Proc. Natl-Acad. Sci., 11: 1037-1041.

Diana, M., Pistis, M., Carboni, S., Gessa, G.L. \& Rossetti, Z.L. (1993) Profound decrement of mesolimbic dopaminergic neuronal activity during ethanol withdrawal syndrome in rats: Electrophysiological and biochemical evidence. Proc. Natl. Acad. Sci., U.S.A., 90: 7966-7969.

Emrich, H.M., Leweke, F.M. y Schneider, U. (1997) Towards a Cannabinoid Hypothesis of Schizophrenia: Cognitive Impairments Due to Dysregulation of the Endogenous Cannabinoid System. Pharmacol. Biochem. \& Behav., 56(4): 803-807.

Fray, P.J.; Dunnett, S.B.; Iversen, S.D.; Björklund, A. y Stenevi, U. (1983): Nigral transplants reinner- 
vating the dopamine-depleted neostriatum can sustain intracranial self-stimulation. Science, 219: 416.

Fredericks, A.B. y Benowitz, N.L. (1980) An abstinence syndrome following.chronic administration of delta-9-tetrahydrocannabinol in rhesus monkeys. Psychopharmacology, 71: 201-202

French, E.D. (1997) $\Delta^{9}$-Tetrahydrocannabinol excites rat VTA dopamine neurons through activation of cannabinoid CB1 but not opioid receptors. Neurosci. Lett, 226: 159-162.

Gaoni, Y. y Mechoulam, R. (1964) Isolation, structure elucidation and partial synthesis of an active constituent of hashish. J. Am. Chem. Soc., 86: 1646-1647.

Gardner, E.L. (1997) Brain reward mechanisms. En: Substance Abuse: A Comprehensive Texbook (Eds.: J.H. Lowinson, P. Ruiz, R.B, Millman \& J.G. Langrod), $3^{\text {a }}$ ed. Williams \& Wilkins, Baltimore, pág: 51-85.

Gardner, E.L. y Lowinson, J.H. (1991) Marijuana's Interaction With Brain Reward Systems: Update 1991. Pharmacol. Biochem. \& Behav., 40: 571-580.

Gardner E.L. \& Vorel, S.R.(1998) Cannabinoid transmission and reward-related events. Neurobiol. Disease, 5: 502-533.

Gueudet, C., Santucci, V., Rinaldi-Carmona, M., Soubrié, P. y Le Fur, G. (1995) The CB1 cannabinoid receptor antagonist SR 141716A affects A9 dopamine neuronal activity in the rat. NeuroReport, 6: 1293-1297.

Jones, R.T., Benowitz, N. \& Bachman, J. (1976) Clinical studies of cannabis tolerance and dependence. Ann. N.Y. Acad. Sci., 282: 221-239.

Johnston, L.D., O'Malley, P.M. \& Bachman, J.G. (1997) Monitoring the future study: 1975-1995: US Dept Health and Human Services. Washington. D.C.

Hall, W., Solowij, N. \& Lemon, J (1994) The health and psychological consequences of cannabis use. Monografía de la serie $n^{\circ} 25$. Australian Goverment Publishing Service. Camberra.

Haney, M., Ward, A.S., Comer, S.D., Foltin, R.W \& Fischman, M.W. (1999) Abstinence symptons following oral THC administration to humans. Psychopharmacology, 141: 385-394.

Herkenham, M. (1992) Cannabinoid Receptor Localization in Brain: Relati: 491-496on-ship to Motor and Reward Systems. Annu.. New York Acad. Sci., 654: 19-32.
Hine, B., Friedman, E., Torrelio, M. \& Gershon, S. (1975) Morphine-dependent rats: Blockade of precipitated abstinence by tetrahydrocannabinol. Science, 187: 443-445.

Hutcheson, D.M., Tzavara, E.T, Smadja, C., Valjent, E., Roques, B.P., Hanoune, J. \& Maldonado, R. (1998) Behavioural and biochemical evidence for signs of abstinence in mice chronically treated with $\Delta$-9-tetrahydrocannabinol. Br. J. Pharmacol., 124.

Koob, G.F.; Sanna, P.P. \& Bloom, F.E. (1998) Neuroscience of Addiction. Neuron, 21: 467-476.

Kouri, E.M., Pope, H.G(Jr.) \& Lukas, S.E. (1999) Changes in aggressive behavior during withdrawal from long-term marijuana use. Psychopharmacology, 143: 302-308.

Laszlo, J., Lucas, V.S., Hanson, D.C., Cronin, C.M. y Sallan, S.E. (1981) Levonantradol for chemotherapy-induced emesis: Phases I-II oral administration. J. Clin. Pharmacol., 21: 51-56.

Ledent, C., Valverde, O., Cossu, G., Petite, F., Aubert, J-F., Beslot, F., Böhme, G.A., Imperato, A., Pedrazzini, T., Roques, B.P., Vassart, G., Fratta, W. y Parmentier, M. (1999) Unresponsiveness to Cannabinoids and Reduced Addictive Effects of Opiates in CB1 Receptor Knockout Mice. Science, 283: 401-404.

Lynn, A.B., Little, M.D., Johnson, M.R., Melvin, L.S., De Costa, B.R. \& Rice, K.C. (1990). Cannabinoid receptor localization in brain. Proc. Natl-Acad. Sci., 87: 1932-1936.

Manzanares, J., Corchero, J., Romero, J., Fernández-Ruiz, Ramos, J.A. \& Fuentes, J.A. (1999) Pharmacological and biochemical interactions between opioids and cannabinoids. TiPS, 20: 287-294.

Martellotta, M.C., Cossu, G., Fattore, G.L., Gessa, G.L. y Fratta, W. (1998) Self-administration of the cannabinoid receptor agonist WIN 55,212-2 in drug naive mice. Neuroscience, 85(2): 327330.

Martín-Calderón, J.L., Muñoz, R.M., Villanúa, M.A, del Arco, I., Moren, J.L., Rodríguez de Fonseca, F. y Navarro, M. (1998) Characterization of the acute endocrine actions of HU-210, a potent synthetic cannabinoid in rats. Eur. J. Pharmacol., , 344: 77-86.

Navarro, M., Fernández-Ruiz, J., de Miguel, R., Hernández, M.L., Cebeira, M. y Ramos, J.A. (1993) An acute dose of $\Delta^{9}$-tetrahydrocannabinol affects behavioral and neurochemical indices of 
mesolimbic dopaminergic activity. Behav. Brain. Res.57: 37-46.

Navarro, M., Hernández, E., Muñoz, R.M., del Arco, I., Villanúa, M.A., Carrera, M.R. y Rodríguez de Fonseca, F. (1997) Acute administration of the CB1 cannabinoid receptor antagonist SR $141716^{a}$ induces anxiety-like responses in the rat. NeuroReport, 8.

Noyes, J.R., Brunk, S.F., Avery, D.H. y Canter, A. (1975) The analgesic properties of delta-9tetrahydrocannabinol and codeine. Clin. Pharmacol., Ther., 18: 84-89.

Olds, J. \& Milner, P. (1954): Positive reinforcement produced by electrical stimulation of septal area and other regions of rat brain. J. Comp. Physiol. Psychol., 47: 419-27.

Piazza, P.V.; Barrot, M.; Rougè-Pont, F. \& al. (1996) Suppression of glucocorticoid secretion and antipsychotic drugs have similar effects on the mesolimbic dopaminergic transmission. Proc. Natl. Acad. Sci. USA, 93: 15445-50.

Piazza, P.V. \& Le Moal, M. (1996) Pathophysiological basis of vulnerability to drug abuse: role of an interaction between stress, glucorticoids and dopaminergic neurons. Annu. Rev. Pharmacol. Toxicol., 36: 359-78.

Poddar, M.K. y Dewey, W.L. (1980) Effects of $\Delta^{9}$ tetrahydrocannabinol on catecholamines uptake and release in hypothalamic and striatal synaptosomes. J. Pharmacol. Exp. Ther. 221: 97-103.

Pope, H.G. (Jr.), Gruber, A.J. \& Yurgelun-Todd, D. (1995) The residual neuropsychological effects of cannabis: the current status of research. Drug Alcohol \& Depend., 38: 25-34.

Raft, D., Gregg, J., Ghia, J. Y Harris, L. (1977) Effects of intravenous tegrahydrocannabinol on experimental and surgical pain. Psychological correlate of the analgesic response. Clin. Pharmacol. Ther., 21: 26-33.

Rodríguez de Fonseca, F., Rubio, P., Menzaghi, F., Merlo-Pich, E., Rivier, J., Koob, G.F y Navarro, M. (1996) Corticotropin-Releasing Factor (CRF) Antagonist [D-Phe $\left.{ }^{12}, \mathrm{Nle}^{21,38}, \mathrm{C}^{\alpha} \mathrm{MeLeu}^{37}\right] \mathrm{CRF}$ Attenuates the Acute Actions of the Highly Potent Cannabinoid Receptor Agonist HU-210 on Defensive-Withdrawal Behavior in Rats. J. Pharmacol.Exp. Ther, 276(1): 56-64.

Rubio, P., Rodríguez de Fonseca, F., Muñoz, R.M., Ariznavarreta, C., Martín-Calderón, J.L. \& Navarro, M. (1995) Long-term behavioral effects of perinatal exposure to $\Delta^{9}$-tetrahydrocannabinol in rats: Possible role of pituitary-adrenal axis. Life Sci., 56(23/24): 2169-2176.

Salamone, J.D, Aberman, J.E, Sokolowsk, J.D. \& Cousins, M.S. Nucleus accumbens dopamine and rate of responding: Neurochemical and behavioral studies. Psychobiology, 27(2): 236247 (1999).

Seth, R. \& Sinha, S. (1991) Chemistry and pharmacology of cannabis. Prog. Drug Res., 36: 71115.

Schultz, W.; Dayan, P. y Montague, P.R. (1997): A neural substrate for prediction and reward. Science, 275: 1593-9.

Tanda, G, Pontieri, F.E. y Di Chiara, G. (1997) Cannabinoid and Heroin Activation of Mesolimbic Dopamine Transmission by a Common M1 Opioid Receptor Mechanism. Science, 276: 248-250.

Vela, G., Ruiz-Gayo, M \& Fuentes, J.A. (1995) Anandamide decreases naloxone-precipitated withdrawal signs in mice chronically treated with morphine. Neuropharmacology, 34: 665-668. 